\title{
Evaluación de la calidad de registro de historias clínicas en consultorios externos del servicio de medicina interna de la Clínica Centenario Peruano Japonesa, 2010-2011
}

Quality assessment of medical records at Clinica Centenario Peruano Japonesa internal medicine doctor's offices, 2010 - 2011

\begin{abstract}
Juan P. Matzumura Kasano, ${ }^{1, a}$, Hugo Gutiérrez Crespo ${ }^{2, b}$, Janeth Sotomayor Salas ${ }^{3, c}$, Gabriela Pajuelo Carrasco ${ }^{4}$

'Profesor Principal, Facultad de Medicina, Universidad Nacional Mayor de San Marcos; Ex Gerente de la Clinica Centenario Peruano Japonesa, Lima, Perú.

${ }^{2}$ Profesor de la Sección Maestría, Facultad de Medicina, Universidad Nacional Mayor de San Marcos; Ex Asistente de Gerencia de la Clínica Centenario Peruano Japonesa, Lima, Perú.

${ }^{3}$ Médico Oftalmóloga, Instituto Nacional de Oftalmología, Lima, Perú. ${ }^{4}$ Estudiante, Facultad de Medicina, Universidad Nacional Mayor de San Marcos, Lima, Perú.

${ }^{a}$ Médico Gineco-Obstetra. Doctor en Medicina; ${ }^{b}$ Obstetra, Magíster en Docencia e Investigación; ${ }^{\circ}$ Maestría en Docencia e Investigación en
\end{abstract}

\begin{abstract}
Resumen
El eje de la auditoria médica es una historia clínica adecuadamente confeccionada. La historia clínica es la constancia escrita de todos los exámenes médicos, estudios realizados y tratamientos aplicados durante el transcurso de la enfermedad. Objetivo: Evaluar la calidad de registro de las historias clínicas de Consultorios Externos del Servicio de Medicina Interna en la Clínica Centenario Peruano Japonesa. Diseño: Descriptivo de corte transversal. Lugar: Consultorios externos del Servicio de Medicina Interna de la Clínica Centenario Peruano Japonesa. Material: Historias clínicas de pacientes atendidos en la consulta externa del Servicio de Medicina Interna. Métodos: Se evaluó 323 historias clínicas mediante un muestreo aleatorio simple, las cuales fueron sometidas a una ficha de auditoria que comprendía 10 ítems, cada uno calificado con 10 puntos, para un total de 100 puntos: fecha y hora de atención, pulcritud y legibilidad, anamnesis adecuada, signos vitales, examen físico, diagnóstico, plan de trabajo, exámenes auxiliares, tratamiento completo y firma y sello del médico. Las historias que obtenían un puntaje total mayor o igual de 80 fueron calificadas como 'aceptables'; las restantes como 'falta mejorar'. Principales medidas de resultados: Historias auditadas aceptables o falta mejorar. Resultados: El 63,8\% (206) de las historias tenía una calidad de registro 'aceptable', mientras que en 36,2\% (117) de historias, la calidad de registro 'falta mejorar'. Los items diagnóstico y tratamiento completo fueron los que presentaron un registro deficiente con mayor frecuencia, tanto en aquellas historias que cumplian un registro aceptable $(64,6 \%$ y $62,6 \%$ llenadas de modo completo, respectivamente) como en aquellas con registro falta mejorar $(20,5 \%$ y $23,1 \%$, respectivamente). La firma y sello del médico tratante fue el ítem que en ambas categorías era el mejor registrado ( $99,5 \%$ y $93,2 \%$, respectivamente). Conclusiones: Más de la mitad de las historias clínicas estudiadas cumplieron con una calidad de registro aceptable para los estándares en la Clínica Centenario Peruano Japonesa.
\end{abstract}

Palabras clave: Auditoria médica, calidad de registro, historias clínicas, consultorios externos.

Abstract

Core of medical audit is a properly tailored medical history. The medical record includes all medical examinations, studies conducted and treatments applied during the course of the disease. Objectives: To assess medical records registration quality of Internal Medicine doctor's offices at Clinica Centenario Peruano Japonesa. Design: Descriptive cross-sectional study. Location: Internal Medicine outpatient offices at Clinica Centenario Peruano Japonesa. Material: Clinical records of patients attended in Internal Medicine doctor's offices. Methods: Three hundred and twenty-three medical records were collected by random sampling and subjected to a 10-items record audit, each rated at 10 points, for a total of 100 points. Items included date and time of care, neatness and legibility suitable record, vital signs, physical examination, diagnosis, plan of work, laboratory findings, complete treatment, and signature and stamp of the physician. Records with total score more than or equal to 80 were rated as 'acceptable', and all others as 'need to improve'. Main outcome measures: 'Acceptable' or 'need to improve' medical records. Results: Two hundred and six (63.8\%) clinical records were rated as 'acceptable' and $117(36.2 \%)$ as 'need to improve'. Items diagnosis and complete treatment presented poor registry more frequently, both in records with acceptable registry (filled in completely in $64.6 \%$ and $62.6 \%$ respectively) and those with need to improve registry (20.5\% and $23.1 \%$ respectively). Signature and seal of the attending physician were the best items in both categories (99.5\% and $93.2 \%$ respectively). Conclusions: More than half of the medical records had an acceptable quality at the Clinica Centenario Peruano Japonesa.

Keywords: Medical audit, quality registration, medical records, outpatient doctor.

An Fac med. 2014;75(3):251-7 / doi: http://dx.doi.org/10.15381/anales.v75i3.9781 


\section{INTRODUCCIÓN}

El presente estudio surgió del interés por evaluar la calidad de registro de las historias clínicas en un área concurrida de una clínica privada, como lo constituyen los consultorios externos del Servicio de Medicina Interna de la Clínica Centenario Peruano Japonesa. La evaluación de la calidad de registro de las historias clínicas es parte de las finalidades de la auditoría médica y de vital importancia, debido que las historias clínicas constituyen documentos de alto valor médico, gerencial, legal y académico. Como tal, la información en las mismas contribuye directa e indirectamente en la calidad de atención a los pacientes, optimiza la gestión de los establecimientos, protege los intereses legales del paciente, del personal de salud y del establecimiento; favorece la investigación y docencia.

Las instituciones de salud del sector privado deben garantizar el cumplimiento de sus objetivos y por tanto su misión. Para esto necesita conocer en qué medida se están logrando estos objetivos para así reconocer las fallas de los procesos que impiden prestar un servicio de calidad. Esta evaluación forma parte de uno de los subprocesos de la administración: el control ${ }^{(1-3)}$. Existen diversos métodos utilizados en el proceso de control; uno de ellos es la auditoría médica. Se ha demostrado que la aplicación de estos métodos (entre ellos la auditoría) ha sido beneficiosa en la mejora de la calidad de atención y el desempeño de los médicos ${ }^{(4-6)}$.

Algunos estudios han demostrado que la implementación de intervenciones para mejorar la calidad de registros bajo un entorno multidisciplinario puede mejorar la práctica profesional, evidenciando un mejor desempeño. Los procesos de auditoría y retroalimentación tienen efectos favorables, sobre todo en el comportamiento; asimismo, su implementación en el área rural puede ser muy limitada y con ello podría generar desigualdades, por lo cual se debería analizar antes de su implementación ${ }^{(7)}$.
Existen diversos estudios que han demostrado que la calidad de las historias clínicas influye directamente en la calidad de atención al paciente. Se ha considerado el buen desarrollo de las historias clínicas como un parámetro para la evaluación de la calidad en la prestación de servicios de salud ${ }^{(8-10)}$. Por otra parte, el acto médico que realiza el profesional tiene como sustento a la historia clínica, la misma que tiene que ser veraz y completa. Asimismo, tiene un extraordinario valor jurídico en los casos de responsabilidad médica profesional, al convertirse por orden judicial en la prueba material principal de todos los procesos de responsabilidad profesional médica, constituyendo un documento médico-legal fundamental y de primer orden ${ }^{(11)}$.

Además la correcta elaboración de las historias clínicas tiene un beneficio económico, especialmente en las instituciones de salud privadas, en donde se exige una evaluación rigurosa y detallada de las historias como requisito para un posible reembolso por parte de las aseguradoras, ya que de no ser así, es la institución de la salud la que debe cubrir el gasto de la intervención ${ }^{(12-14)}$.

El objetivo del presente trabajo fue evaluar la calidad de registro de las historias clínicas de Consultorios Externos del Servicio de Medicina Interna en la Clínica Centenario Peruano Japonesa, durante los años 2010 - 2011.

\section{MÉTODOS}

El estudio fue de tipo descriptivo, no experimental, de corte transversal y retrospectivo. El ámbito de investigación comprendió los Consultorios Externos del Servicio de Medicina Interna de la Clínica Centenario Peruano Japonesa.

El material de estudio estuvo conformado por el total de historias clínicas correspondientes a los pacientes nuevos de ambos sexos que acudieron al consultorio externo del Servicio de Medicina Interna de la Clínica Centenario Peruano Japonesa. Los criterios de inclusión fueron los pacientes nuevos mayores de
18 años atendidos en los consultorios externos del Servicio de Medicina Interna durante el período de estudio. Se excluyó los pacientes nuevos no atendidos en los consultorios externos del Servicio de Medicina Interna, historias clínicas de pacientes que estuvieran en procesos médico-legales, historias solicitadas para informe médico u otra auditoria médica, historias clínicas que no se encontraran dentro de los ambientes de archivos clínicos en el momento de la evaluación.

Para la estimación del número total de historias, se obtuvo información del Departamento de Archivo de la Clínica. Se obtuvo un marco muestral total de 3870 historias clínicas de consultorio externo del Servicio de Medicina Interna. Se consideró el máximo tamaño muestral posible basándonos en la fórmula presentada por la Norma Técnica de Auditoría de Prestaciones de Salud en el Seguro Social de Salud - EsSalud $\left(\mathrm{N}=\mathrm{Z}^{2 *} \mathrm{p} * \mathrm{q} * \mathrm{~N} /\left[\mathrm{e}^{2}\right.\right.$ $\left.*(\mathrm{~N}-1)]+\left[\mathrm{Z}^{2 *} \mathrm{p} * \mathrm{q}\right]\right)=350$ historias clínicas, donde $\mathrm{Z}$ es la confiabilidad al 95\% (1,96), p es 0,5 (asumiendo que $50 \%$ del registro estará llenado correctamente, dado que no contamos con prevalencias obtenidas de estudios similares realizados en clínicas privadas), q es (1- p), $\mathrm{N}$ es el número de historias clínicas totales realizadas en el periodo citado, que corresponde a 3870 historias clínicas y error de precisión (considerado 0,05). El tamaño de la muestra fue 350 historias clínicas. Para la selección de las unidades de análisis se usó el muestreo probabilístico de tipo aleatorio simple.

Se seleccionó 350 historias clínicas con la autorización del departamento de Archivo de la Clínica. Previamente, se realizó una prueba piloto de 10 historias clínicas auditadas, no incluidas en la muestra total. Se elaboró una ficha de auditoría de historia clínica de Consultorio Externo de la Clínica Centenario Peruano Japonesa, la misma que fue adaptada de la Norma Técnica de elaboración de Historia Clínica del Ministerio de Salud y utilizado por la institución desde el año 2008, median- 
te una prueba de consistencia interna aplicando Kuder y Richardson (KR$20)$, cuya puntuación obtenida correspondió 0.84 .

La ficha constó de dos partes; la primera recogió datos para identificar la historia clínica, el paciente y el médico tratante; firma, sello, fecha y hora de consulta. La segunda recogió información sobre la calidad de la historia mediante 10 ítems, a los cuales se le asignó la puntuación de 10 si estaban presentes, 5 si estaban incompletos y 0 si no estaban presentes. Los ítems fueron pulcritud, legibilidad, anamnesis, antecedentes, signos vitales, examen físico, plan de trabajo, exámenes auxiliares, diagnóstico y tratamiento. La puntuación final clasificó la variable calidad de registro de la historia clínica en dos categorías: 'aceptable' si el puntaje era mayor o igual a 80 , y 'falta mejorar' si dicho puntaje era menor a 80 , tomando como elementos de sustento la escala valorativa de la norma técnica para calidad de registro de historia clínica aprobada por el Ministerio de Salud y teniendo en cuenta la disposiciones aprobadas por las autoridades de la Clínica Centenario Peruano Japonesa a partir del año 2009.

\section{RESULTADOS}

Del total de 350 historias clínicas calculadas para la muestra, 19 no fueron halladas en el archivo en el momento de la auditoría. Se revisó un total de 331 historias, de las cuales 8 fueron excluidas del análisis, 6 de ellas por no presentar consulta alguna en el servicio de medicina interna y 2 por estar en blanco. Finalmente, un total de 323 historias fueron auditadas correctamente. De ellas, 63,8\% (206) tuvieron una calidad de registro 'aceptable', mientras que en $36,2 \%$ (117) de historias la calidad de registro 'falta mejorar'. El porcentaje de historias clínicas con calidad de registro calificada como 'aceptable' estuvo entre $50 \%$ y $80 \%$, donde el mínimo porcentaje $(50 \%)$ fue ubicado en los meses de mayo y noviembre de 2010 y los meses de mayor porcentaje
Tabla 1. Calidad de registro según mes de consulta de las historias clínicas.

\begin{tabular}{cccc} 
Mes de consulta & \multicolumn{2}{c}{ Calidad de registro } & Total \\
\cline { 2 - 3 } Abril 2010 & Falta mejorar (\%) & Aceptable $(\%)$ & 15 \\
Mayo 2010 & $5(33 \%)$ & $10(67 \%)$ & 16 \\
Junio 2010 & $8(50 \%)$ & $8(50 \%)$ & 15 \\
Julio 2010 & $3(20 \%)$ & $12(80 \%)$ & 16 \\
Agosto 2010 & $6(38 \%)$ & $10(62 \%)$ & 23 \\
Septiembre 2010 & $7(30 \%)$ & $16(70 \%)$ & 24 \\
Octubre 2010 & $8(33 \%)$ & $16(67 \%)$ & 35 \\
Noviembre 2010 & $17(49 \%)$ & $18(51 \%)$ & 32 \\
Diciembre 2010 & $16(50 \%)$ & $16(50 \%)$ & 26 \\
Enero 2011 & $12(46 \%)$ & $14(54 \%)$ & 28 \\
Febrero 2011 & $6(21 \%)$ & $22(79 \%)$ & 35 \\
Marzo 2011 & $11(31 \%)$ & $24(69 \%)$ & 31 \\
Abril 2011 & $7(23 \%)$ & $24(77 \%)$ & 27 \\
\hline Total & $11(41 \%)$ & $16(59 \%)$ & 323 \\
\hline
\end{tabular}

de calidad de registro 'aceptable' fueron junio de 2010 (80\%) y enero de 2011 (79\%). Asimismo, el porcentaje de historias clínicas calificadas como una calidad de registro que 'falta mejorar' se ubicó entre $20 \%$ y $50 \%$, presentando sus máximos porcentajes precisamente en los meses de mayo y noviembre de 2010, tal como se observa en la tabla 1.
El gráfico 1 muestra la media del puntaje total asignado a las historias clínicas pertenecientes al período estudiado; se mantuvo dentro del rango de 77 y 87,33 puntos. Se observó que el mes de abril de 2010 correspondió al periodo donde la media del puntaje total alcanzado fue menor, mientras que el mes en el que la media fue mayor correspondió a junio de 2010.

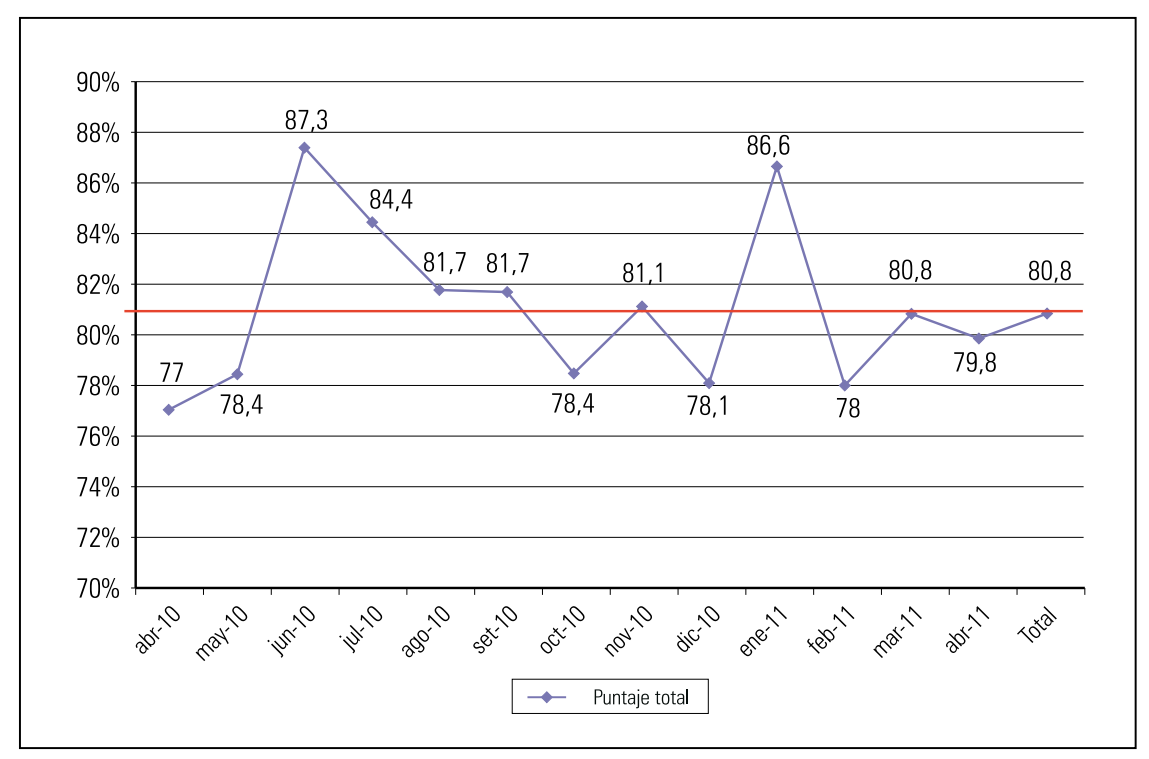

Gráfico 1. Puntaje total según mes de consulta de las historias clínicas. 
De las diferentes variables analizadas, los indicadores que se encontraron completos en más de $70 \%$ de las historias clínicas fueron la firma y sello, presentes en $97,2 \%$, y los exámenes auxiliares, en $73,1 \%$. La fecha y hora de consulta constaban en $59,1 \%$ de historias clínicas y un $65,9 \%$ fue calificado como pulcra y legible. La anamnesis y los antecedentes estuvieron incluidos en $61,6 \%$. Tres o más signos vitales fueron consignados en $65,6 \%$. El examen físico general y regional se encontró en $63,8 \%$, el plan de trabajo adecuado en $62,5 \%$.

El diagnóstico, incluido el CIE-10, solo figuraba en $48.6 \%$ y el tratamiento completo en $48,3 \%$, registrando los más bajos porcentajes de la calidad de registro completa. El único indicador que se encontró incompleto en más del 50\% de historias clínicas fue precisamente el de diagnóstico.

De las 206 historias clínicas calificadas como aceptables, los indicadores que fueron registrados de forma completa con mayor frecuencia fueron la firma y el sello del médico tratante $(99,5 \%)$, seguido de los exámenes auxiliares pertinentes $(88,3 \%)$ y en un tercer lugar el plan de trabajo adecuado $(82,5 \%)$. Precisamente, el indicador registrado de forma incompleta con mayor frecuencia fue el diagnóstico $(35,4 \%)$ seguido del registro de signos vitales $(27,7 \%)$. Solo 1,9\% de historias clínicas 'aceptables' no solicitaron exámenes auxiliares pese a haber sido pertinente su indicación, tal como aparece en la tabla 2.

En la tabla 3 se puede observar que de las 117 historias clínicas cuya calidad de registro falta mejorar, los indicadores con mayor subregistro fueron el diagnóstico, que en $76,9 \%$ de las historias clínicas se auditó incompleto, seguido por el examen físico y el tratamiento completo, que en $55,6 \%$ de las historias clínicas no fue registrado de forma completa. Todos los indicadores de registro en las historias clínicas calificadas como 'falta mejorar' tuvieron por lo menos una historia clínica en la que no se consignó o no se cumplió con

Tabla 2. Frecuencia de registro de los indicadores de las historias clínicas 'aceptables'.

\begin{tabular}{cccc} 
Indicadores de registro & \multicolumn{3}{c}{ Calidad de registro } \\
\cline { 2 - 4 } Fecha y hora de la atención & $0(0,0 \%)$ & $66(32,0 \%)$ & $140(68,0 \%)$ \\
Pulcritud y legibilidad & $0(0,0 \%)$ & $43(20,9 \%)$ & $163(79,1 \%)$ \\
Anamnesis adecuada con antecedentes & $0(0,0 \%)$ & $49(23,8 \%)$ & $157(76,2 \%)$ \\
Signos vitales & $1(0,5 \%)$ & $57(27,7 \%)$ & $148(71,8 \%)$ \\
Examen físico & $1(0,5 \%)$ & $44(21,4 \%)$ & $161(78,2 \%)$ \\
Diagnóstico & $0(0,0 \%)$ & $73(35,4 \%)$ & $133(64,6 \%)$ \\
Plan de trabajo & $0(0,0 \%)$ & $36(17,5 \%)$ & $170(82,5 \%)$ \\
Exámenes auxiliares & $4(1,9 \%)$ & $20(9,7 \%)$ & $182(88,3 \%)$ \\
Tratamiento completo & $1(0,5 \%)$ & $76(36,9 \%)$ & $129(62,6 \%)$ \\
Firma y sello del médico tratante & $0(0,0 \%)$ & $1(0,5 \%)$ & $205(99,5 \%)$ \\
\hline
\end{tabular}

Tabla 3. Frecuencia de registro de los indicadores de las historias clínicas 'falta mejorar'.

\begin{tabular}{cccc}
\multirow{2}{*}{ Indicadores de registro } & \multicolumn{3}{c}{ Calidad de registro } \\
\cline { 2 - 4 } Fecha y hora de la atención & $2(1,7 \%)$ & $64(54,7 \%)$ & $51(43,6 \%)$ \\
Pulcritud y legibilidad & $1(0,9 \%)$ & $66(56,4 \%)$ & $50(42,7 \%)$ \\
Anamnesis adecuada con antecedentes & $2(1,7 \%)$ & $73(62,4 \%)$ & $42(35,9 \%)$ \\
Signos vitales & $1(0,9 \%)$ & $52(44,4 \%)$ & $64(54,7 \%)$ \\
Examen físico & $7(6,0 \%)$ & $65(55,6 \%)$ & $45(38,5 \%)$ \\
Diagnóstico & $3(2,6 \%)$ & $90(76,9 \%)$ & $24(20,5 \%)$ \\
Plan de trabajo & $23(19,7 \%)$ & $62(53,0 \%)$ & $32(27,4 \%)$ \\
Exámenes auxiliares & $23(19,7 \%)$ & $40(34,2 \%)$ & $54(46,2 \%)$ \\
Tratamiento completo & $25(21,4 \%)$ & $65(55,6 \%)$ & $27(23,1 \%)$ \\
Firma y sello del médico tratante & $3(2,6 \%)$ & $5(4,3 \%)$ & $109(93,2 \%)$ \\
\hline
\end{tabular}

el indicador requerido, siendo tanto el plan de trabajo $(19,7 \%)$, los exámenes auxiliares $(19,7 \%)$ y el tratamiento completo aquellos indicadores con mayor ausencia de registro. En estas historias clínicas solo el registro de 3 o más signos vitales superó el llenado completo en más de la mitad (54,7\%).

\section{DISCUSIÓN}

Los hallazgos de la auditoría en la calidad de registro de las 323 historias clínicas dieron como resultado un puntaje total de 80,79 , promedio considerado como 'aceptable'. Si bien lo obtenido fue satisfactorio, se debe tener en cuenta que 117 (36,2\%) historias clínicas estaban en el rubro 'por mejorar'.
El estudio de Bocanegra y col. evaluó el nivel de calidad de registro de historias clínicas de pacientes hospitalizados en un hospital de Trujillo, mediante una ficha de verificación de calidad de las mismas, elaborada en base a la Norma Técnica de Salud para la Gestión de la Historia Clínica. Se concluyó que el nivel de calidad del registro de la mayor parte de los ítems de la mayoría de los formatos de las historias clínicas de hospitalización de los pacientes fue deficiente; asimismo, que el nivel de calidad del registro de la mayoría de las mencionadas historias era regular y que el de las restantes era bueno. Es importante señalar que en la Clínica donde se desarrolló nuestra investigación, la auditoría es un procedimiento rutinario ${ }^{(11)}$. 
Del total de historias auditadas se observa que el ítem de fecha y hora no es consignado de manera adecuada en 130 historias clínicas $(40,2 \%)$ y que solo se encontró 2 historias sin este ítem, siendo uno de las recomendaciones más fáciles de cumplir, que de acuerdo con la norma técnica del MINSA para las historias clínicas debería ser consignada en toda consulta ambulatoria. Llanos y col. realizaron una auditoria médica de historias clínicas de consulta externa en cuatro hospitales del Perú y hallaron que el llenado de las historias clínicas es bastante pobre para fecha y hora de consulta $(13,7 \%)^{(12)}$.

En el estudio de Conesa y col. se analizó informes de asistencia a urgencias de 6 hospitales de Barcelona y resultó que $16 \%$ de los informes presentaba un contenido informativo insuficiente y/o eran prácticamente ilegibles, por lo que planteaban dificultades importantes en la confección del alta ${ }^{(29)}$. En el trabajo de Castillo y col. sobre la evaluación estadística de la calidad de los registros de salud se concluye que la falta de datos clínicos o la ilegibilidad, no importa que tan grande o pequeño sea ese porcentaje, debe llamar a la meditación e interpelar a la conciencia general sobre las implicaciones de los registros incompletos y mal estructurados en la calidad de la atención y la buena utilización de los recursos económicos y humanos con que cuentan los prestadores de servicios de salud. Cabe mencionar que englobar anamnesis y antecedentes en un solo ítem no permite un correcto análisis de cada uno; por ello, en los instructivos de la norma técnica del MINSA se consignan por separado ${ }^{(30)}$.

Con respecto a los signos vitales, se encontró el cumplimiento adecuado en 66\% de las historias clínicas. Es de resaltar que no se consigna en muchas de las historias la temperatura de los pacientes, incluso si el diagnóstico era de tipo infeccioso. Cabe destacar que en el instructivo del MINSA los signos vitales se encuentran dentro del examen clínico y se consigna frecuencia cardiaca y presión arterial como los signos bases a los que se pueden agregar los demás signos, dependiendo de la patología. Caso contrario a nuestro trabajo en el ítem de signos vitales es el resultado de la investigación de Llanos y col., que consistió en la auditoría de las historias clínicas en consulta externa de 4 hospitales peruanos, donde se encontró el registro de funciones vitales en solo $8,75 \%$ de todas las historias ${ }^{(12)}$.

En cuanto al examen físico, se obtuvo un $64 \%$ de historias correctamente llenadas, 34\% a medias y 8 historias sin examen físico alguno. En el caso de tratarse de una patología que no necesitaba de un exhaustivo examen físico, se otorgaba el puntaje máximo con la presencia del examen regional orientado. Los resultados encontrados en este estudio contrastan con la investigación de Cordero en un centro médico de EsSalud con sistema de gestión hospitalaria, que registra 92\% (230) de historias clínicas sin examen general y $24,8 \%$ (62) sin examen regional ${ }^{(32)}$.

Con respecto al diagnóstico, se observa que del total de historias clínicas evaluadas solo 157 historias (48,6\%) lo completaron correctamente, es decir que colocaron el diagnóstico y el código CIE correspondiente; 163 (50,5\%) tuvieron llenado incompleto (diagnóstico o código CIE) y solo en 3 historias clínicas (9\%) no se encontró ninguno de los dos ítems. Por otro lado, se observó que la mayoría de historias clínicas calificadas como incompletas en este rubro solo presentaban el diagnóstico clínico y no tenían el código CIE. En un estudio de Carrasco y col. se revisó un total de 3261 historias clínicas de consultorio externo, de las cuales 80\% tenía registrado el diagnóstico de la consulta ${ }^{(33)}$. Por otra parte, Pestana y col. revisaron un total de 384 formatos de atención utilizados en diferentes especialidades del servicio de Pediatría, de los cuales 5,46\% (21 formatos) no registró el diagnóstico. El objetivo del estudio de Pestana fue determinar la compatibilidad del diagnóstico registrado por los médicos con los códigos asignados por el personal de estadística de acuerdo al CIE 10, encontrando un valor de $49.2 \%$ de compatibilidad ${ }^{(34)}$.
Estos datos demuestran la importancia de que el código CIE 10 sea registrado junto con el diagnóstico por los mismos doctores. El plan de trabajo fue registrado de manera adecuada en $62,5 \%$ (202) de las historias clínicas revisadas. Estos hallazgos también difieren por los reportados por autores como Perata y col., que encontraron el registro de este criterio en $84,6 \%$ de la muestra evaluada ${ }^{(25)}$.

En cuanto a los exámenes auxiliares, estos fueron registrados en 73,1\% (236) del total de historias clínicas revisadas. No se encontró valores comparables con respecto al registro de exámenes auxiliares en otros estudios, puesto que gran parte de ellos considera los exámenes auxiliares como parte del plan de

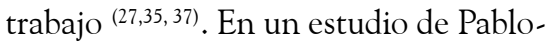
Rocano solo se evaluó el registro de los resultados de los exámenes auxiliares o el comentario y/o apreciación de los mismos, el cual fue $50 \%$ de la muestra ${ }^{(10)}$.

Con respecto al tratamiento, se observó que 48,3\% (156) de historias auditadas cumplió con los criterios de un tratamiento adecuado, es decir que, consignaron al menos el nombre genérico, dosis, vía de administración y frecuencia del fármaco; gran parte del $43,7 \%$ de historias clínicas que fueron calificadas con un tratamiento inadecuado fue debido a la ausencia de alguno de estos criterios, sobretodo de la vía de administración y frecuencia de consumo del fármaco. Comparado con nuestros resultados, los obtenidos por González y col. mostraron un cumplimiento adecuado de este criterio en $87,1 \%$ de su muestra ${ }^{(36)}$; un resultado similar fue hallado por Bocanegra y col., con $91,4 \%$ de historias clínicas con registro explícito del tratamiento aplicado ${ }^{(11)}$. Por el contrario, en el estudio de Perata y col. se encontró que el tratamiento farmacológico se registró completamente en 7,4\% de la muestra estudiada ${ }^{(25)}$. La diferencia de los valores obtenidos en los diferentes estudios y también en el nuestro puede obedecer a los criterios de análisis utilizados para la categorización de este ítem como 
completo (adecuado) o incompleto (inadecuado); por otro lado, algunos estudios consideran el tratamiento como parte del plan de trabajo o lo evalúan en conjunto ${ }^{(12,33)}$.

La consigna de la firma y sello del médico tratante fue encontrada en 97,2\% (314) de las historias clínicas evaluadas. Estos resultados difieren enormemente a los obtenidos en los estudios de Perata y col. y Llanos-Mayca y col., que obtuvieron resultados de $49,7 \%$ y $54,7 \%$, respectivamente ${ }^{(12,25)}$. En general, se ha encontrado que las categorías con porcentajes más bajos de registro completo son la de Diagnóstico y Tratamiento (48,6\% y 48,3\%, respectivamente). Esta distribución se mantiene cuando se evalúa las categorías agrupando las historias clínicas en grupo de 'aceptables' (puntaje $\geq 80$ ) y 'falta mejorar' (puntaje < 80). En el primer grupo se observó que en todos los criterios el porcentaje de cumplimiento es mayor de $60 \%$, encontrándose los valores más bajos en las categorías de diagnóstico y tratamiento. Por otra parte, en el grupo de historias clínicas calificadas como 'falta mejorar' se observó que en la gran mayoría de criterios (excepto el de firma y sello) el porcentaje de cumplimiento no llegó a alcanzar el $55 \%$, encontrándose también las menores tasas de registro completo en las categorías de diagnóstico y tratamiento (20,5\% y $23,1 \%$, respectivamente). En contraste, el estudio de Perata y col. obtuvo sus valores más bajos en los rubros de signos vitales y tratamiento $(<20 \%$ y $7,4 \%$, respectivamente) ${ }^{(27)}$. Así también, en el estudio de Llanos-Zavalaga y col. las categorías con menor porcentaje de registro fueron el de los signos vitales y de fecha y hora de atención $\left(8,75 \%\right.$ y $13,7 \%$, respectivamente) ${ }^{(12)}$.

Se concluye que más de la mitad de las historias clínicas de los consultorios de Medicina Interna cumplieron con una calidad de registro aceptable para los estándares establecidos por la clínica. Los ítems de diagnóstico y tratamiento completo suelen ser los que presentaron con mayor frecuencia un registro deficiente. La firma y sello del médico tratante es el ítem que en ambas categorías fue el mejor registrado en el presente estudio.

\section{AGRADECIMIENTOS}

Un agradecimiento a los directivos de la Clínica Centenario Peruano Japonesa, al personal de historias clínicas, por el apoyo brindado durante el desarrollo de la presente investigación.

\section{REFERENCIAS BIBLIOGRÁFICAS}

1. Stoner J, Freeman R, Gilbert D. Administración. 6ta Edición. México. 1996.

2. Houghton G. From audit to effectiveness: an historical evaluation of the changing role of Medical Audit Advisory Groups. J Eval Clin Pract. 1997 Nov;3(4):245-53.

3. Scales D, Dainty K, Hales B, Pinto R, Fowler RA, Adhikari NK, Zwarenstein M. A multifaceted intervention for quality improvement in a network of intensive care units: a cluster randomized trial. JAMA. 2011 Jam 26;305(4):363-72. doi: 10.1001/ jama.2010.2000.

4. Vodstrcil LA, Hocking JS, Cummings R, Chen MY, Bradshaw CS, Read TR, Sze JK, Fairley CK. Computer assisted self interviewing in a sexual health clinic as part of routine clinical care; impact on service and patient and clinician views. PloS One. 2011 Mar;6(3):1-9. doi: 10.1371/journal. pone.0018456.

5. Buitrago F, Guillén J, Mentía JM, Agudo B, García M, Molina LJ. Análisis y evolución de la calidad de las Historias Clínicas en un Centro de Salud. Rev San Hig Púb. 1991;65(5):421-7.

6. Hartig JR, Allison J. Physician performance improvement: an overview of methodologies. Clin Exp Rheumatol. 2007 Nov-Dec;25(6 Suppl 47):50-4.

7. Althabe F, Bergel E, Cafferata ML, Gibbons L, Ciapponi A, Alemán A, Colantonio L, Palacios AR. Strategies for improving the quality of health care in maternal and child health in low- and middle-income countries: an overview of systematic reviews. Paediatr Perinat Epidemiol. 2008 Jan;22 Suppl 1:42-60. doi: 10.1111/j.1365-3016.2007.00912.x.

8. Carpintero JM, Ochoa FJ, Acítores JM, Ferrús JA, Fernández JA. Grado de cumplimentación de la Historia Clínica de Urgencias como indicadores de la Calidad Asistencial. Emergencias. 1997; 9 (1): $26-30$.

9. De Miguel B, Santiago A, Candia B, Blanco A, Muñoz D, Martín M ${ }^{\mathrm{T}}$, Jiménez J, González T, Rodríguez C. La historia clínica de urgencias. Un parámetro de la Calidad de la Asistencia. Emergencias. 1997;9(1):31-4.

10. Rocano EP. Evaluación de la calidad de los registros de las historias clinicas de los pacientes fallecidos en el Servicio de Emergencia de un Hospital General. Rev Soc Peru Med Int. 2008;21(2):51-4.

11. Bocanegra S, Bocanegra G; Alvarado V. Nivel de calidad del registro de las historias clinicas de pacientes apendicectomizados en el Hospital Belén de Trujillo de enero a junio de 2007. Rev Med Vallejiana. 2008;5(2):115 -24.
12. Llanos LF, Mayca J, Navarro GC. Auditoría médica de historias clínicas en consulta externa de cuatro hospitales públicos peruanos. Rev Med Hered. 2006;17(4):220-6.

13. Ali SA, Soomro AG, Tahir SM, Memon AS. Prospective basic clinical audit using minimal clinical data set. J Ayub Med Coll Abbottabad. 2010 JanMar;22(1):58-61.

14. Garaycochea V. Auditoria Médica. Pediátrica. 2000;3(1):27-8.

15. Fonseca LJA, Rivero SE, Baltazar TJA, Ñamendys SSA, Domínguez CG. Auditoría médica. Med Int Mex. 2009;25(1):23-30.

16. Piscoya J. Calidad de la atención en salud a través de la auditoría médica. An Fac med. 2000;61(3):227-40.

17. Ministerio de Salud del Perú. Norma técnica Peruana de auditoría en salud para el mejoramiento de la calidad de los servicios. [en linea] 2011 [fecha de acceso 3 de mayo de 2011]. Disponible en: http://www.minsa.gob.pe/portada/docconsulta2004.asp.

18. Diario Oficial El Peruano. 2005. 29 de junio; página 32 .

19. Jamtvedt G, Young JM, Kristoffersen DT, O'Brien MA, Oxman AD. Audit and feedback: effects on professional practice and health care outcomes. Cochrane Database Syst Rev. 2006 Apr 19;(2):CD000259.

20. Llanos F. Auditoría médica en el primer nivel de atención. Rev Med Hered: 2000; 11: 107-112.

21. Hardman E, Joughin C. Clinical audit: what it is and what it isn't. En: Focus on Clinical Audit in Child and Adolescent Mental Health Services. London: Gaskell; 1998:3-6.

22. European Commission. Radiation protection. European Commission guidelines on Clinical audit for medical radiological Practices. Diagnostic Radiology, Nuclear Medicine and Radiotherapy [update 2009 Junio 9; citado 2011].

23. Selman L, Harding R. How can we improve outcomes for patients and families under palliative care? Implementing clinical audit for quality improvements in resource limited settings. Indian J Palliat Care. 2010 Jan;16(1):8-15. doi: 10.4103/09731075.63128.

24. Donabedian A. Evaluating the quality of medical care. Millbank Memorial Fund Quarterly. 1996;44:166-206.

25. Perata - Salazar M, Llanos - Zavalaga LF, Cabello Morales EA, Mayca Pérez J. Auditoría médica en la consulta externa pediátrica en un hospital general, Lima-Perú. Rev Med Hered. 2006;17(1):35-41.

26. Ministerio de Salud. Norma técnica de la historia clínica de los establecimientos del sector salud. Perú [en línea] 2010 [fecha de acceso 14 de junio de 2011]. Disponible en htpp://www.minsa.gob.pe/ normaslegales /2005.pdf.

27. Ministerio de Salud. Norma técnica de auditoría de de la calidad de atención en salud. Perú 2005. [en línea] 2005 [fecha de acceso 7 junio de 2010]. Disponible en htpp://www.minsa.gob.pe/normaslegales /2005.pdf.

28. Seguro Social de Salud. Normas y Procedimientos para la auditoría médica de prestaciones de salud en el Seguro Social de Salud (ESSALUD) Lima, 2010.

29. Conesa A, Muñoz R, Torre P, Gelabert G, Casanellas JM, Trilla A, Asenjo MA. Evaluación de los informes de asistencia de urgencias como instrumento de gestión de la información clínica. Med Clin (Barc). 2003;120(19):734-6. 
30. Castillo J. Registros de salud: evaluación estadística de su calidad. Rev Scienc Adm Financ Segur Soc. 1997;5(2):17-21.

31. López-Picazo JJ, Agulló F, Villaescusa M, Cerezo JM. Datos clínicos esenciales de la historia clínica de atención primaria: una experiencia de evaluación y mejora. Aten Primaria. 2002;30(2):92-8.

32. Cordero RC. Auditoria Médica en un Centro Médico de ESSALUD con Sistema de Gestión Hospitalaria [tesis]. Lima: Universidad Nacional Mayor de San Marcos; 2002.

33. Carrasco M, Mazzi E. Auditoria médica en consultorio externo de Pediatria. Rev Soc Bol Ped. 1993;32(1):20-4.
34. Pestana R, Llanos LF, Cabello EA, Lecca L. Concordancia entre el diagnóstico médico y la codificación de informática, considerando el CIE-10, en la consulta externa de pediatría en el Hospita Nacional Cayetano Heredia, Lima-Perú. Rev Med Hered. 2005;16(4):239-45.

35. González J, Areces M, Marcelo S, Cabulli D, Iglesias L. Calidad de las historias clínicas: un camino hacia la mejora de la comunicación institucional. [en línea]. 2011 [fecha de acceso 23 de junio de 2011]. Disponible en: http://www.ramos mejia.org. ar minsa.gob.pe/portada/docconsulta2004.asp.
Artículo recibido el 13 de enero de 2014 y aceptado para publicación el 29 de junio de 2014.

Conflictos de interés:

El Dr. Juan Matzumura Kasano y el Dr. Hugo Gutiérrez Crespo son ex directivos de la Clínica Centenario Peruano Japonesa.

\section{Correspondencia:}

Juan P. Matzumura Kasano

Av. Javier Prado 1066 Piso 7- San Isidro

Correo electrónico; jmatzumura@yahoo.com 